Pacific Journal of Mathematics

ON A CLASS OF SINGULAR SECOND ORDER DIFFERENTIAL
EQUATIONS WITH A NON LINEAR PARAMETER 


\section{ON A CLASS OF SINGULAR SECOND ORDER DIFFERENTIAL EQUATIONS WITH A NON LINEAR PARAMETER}

B. W. Roos

Introduction. Singular second order differential equations with a non-linear parameter play an important role in mathematical physics. For instance, the radial wave equation for a relativistic particle with zero spin in a centrally symmetric potential field, $V(r)$, the KleinGordon equation:

$$
\begin{aligned}
u^{\prime \prime}+\frac{2}{r} u^{\prime}+\left\{\left[\frac{E+e V(r)}{\hbar c}\right]^{2}-\frac{l(l+1)}{r^{2}}-\left(\frac{M c}{\hbar}\right)^{2}\right\} u & =0 \\
& {\left[^{\prime} \equiv d / d r\right] }
\end{aligned}
$$

contains the energy parameter $E$ in a nonlinear fashion. If $V(r)=$ $a Z / r$ is a Coulomb field the transformation $x=2\left(1-E^{2}\right)^{1 / 2} r$ will transform equation (1) into a Whittaker equation

$$
\frac{d^{2} u}{d x^{2}}+\left[-\frac{1}{4}+\frac{k}{x}+\frac{1 / 4-m^{2}}{x^{2}}\right] u=0
$$

where

$$
k=E \alpha Z\left(1-E^{2}\right)^{-1 / 2}
$$

and

$$
m=\left[(l+1 / 2)^{2}-\alpha^{2} Z^{2}\right]^{1 / 2} .
$$

The eigenvalues are the roots of a transcendental equation [1]

$$
\begin{gathered}
\frac{B-n \pi}{\lambda}-\frac{1}{\lambda} \arg \left[\Gamma\left(\frac{1}{2}-\frac{E \alpha Z}{\left(1-E^{2}\right)^{1 / 2}}\right)\right. \\
\left.=i\left(\alpha^{2} Z^{2}-(l+1 / 2)^{2}\right)^{1 / 2}\right] \\
=\log \left(1-E^{2}\right)^{1 / 2} .
\end{gathered}
$$

For more general potentials than the Coulomb potential the spectral properties of a second order differential equation with a non-linear parameter are more difficult to obtain. For second order equations with a linear parameter the analytical methods of Weyl [7] and Titchmarsh [5] for the Schrödinger equation

$$
x^{\prime \prime}(r)+[\lambda-\cup(r)] x(r)=0
$$

may be used. These methods have been extended by Titchmarsh [6],

Received April, 11, 1963. 
Sangren and the author $[2,3,4]$ to the investigation of the spectral properties and related expansion theorems of a system of two first order differential equations

$$
\begin{aligned}
& x_{1}^{\prime}(r)-[\lambda a(r)+b(r)] x_{2}(r)=0 \\
& x_{2}^{\prime}(r)+[\lambda c(r)+d(r)] x_{1}(r)=0
\end{aligned}
$$

with singular coefficients. The class of equations of the form (4) is very wide and includes, for instance, not only the non-relativistic radial Schrödinger equation but also the relativistic radial Dirac equations [3]. More examples may be cited of second order differential equations which may be conveniently discussed in terms of the system (4). This is not altogether surprising since in the process of deriving a mathematical model for a physical phenomenon some second order differential equations are actually derived from a system of first order equations.

In this paper we propose to investigate the asymptotic properties of a class of singular second order equations with a non-linear parameter by the simple expediency of reducing the second order equation to a system of equations of the form (4). We will illustrate our discussion by proving a spectral theorem for a member of this subclass:

$$
x^{\prime \prime}(r)+f(r) x^{\prime}(r)+\left[\lambda^{2} g(r)+\lambda h(r)+k(r)\right] x(r)=0 .
$$

The arguments will be presented for the case of a semi-infinite interval $[0, \infty)$ where the point $r=0$ is assumed to be a regular point and the equation is only singular for $r \rightarrow \infty$. The coefficients $f, g, h$ and $k$ are assumed to be real valued continuous functions of $r$ and we will impose the initial condition

$$
p^{-1}(0) \cos \beta x(0)+p(0)(\lambda+1)^{-1} \sin \beta x^{\prime}(0)=0
$$

where $\beta$ is a real parameter and $p$ a function defined in section (4). For $\beta=0$ the initial condition reduces to the condition $x(0)=0$ at $\boldsymbol{r}=0$.

We transform the second order equation into a system of the form (4) and proof a spectral theorem for this system. An equivalent theorem for equation (5) may be deduced from this theorem.

2. Transformation of (5) into (4). Consider the system of equations

$$
\begin{aligned}
& x_{1}^{\prime}(r)-[\lambda a(r)+b(r)] x_{2}(r)=0 \\
& x_{2}^{\prime}(r)+[\lambda c(r)+d(r)] x_{1}(r)=0 .
\end{aligned}
$$

Differentiating the first equation with respect to $r$ and making appropriate substitutions from the second equation, we obtain 


$$
x_{1}^{\prime \prime}(r)-\frac{\lambda a^{\prime}(r)+b^{\prime}(r)}{\lambda a(r)+b(r)} x_{1}^{\prime}(r)+(\lambda a+b)(\lambda c+d) x_{1}(r)=0 .
$$

Equation (7) is equivalent to equation (3) if the following identifications are made:

$$
\begin{aligned}
x_{1}(r) & \equiv x(r) \\
a(r) & =a(0) \exp \left[-\int_{0}^{r} f(r) d r\right] \\
b(r) & =b(0) \exp \left[-\int_{0}^{r} f(r) d r\right] \\
c(r) & =a^{-1}(0) g(r) \exp \left[\int_{0}^{r} f(r) d r\right] \\
d(r) & =b^{-1}(0) k(r) \exp \left[\int_{0}^{r} f(r) d r\right]
\end{aligned}
$$

and the condition

$$
h(r)=a(0) b^{-1}(0) k(r)+b(0) a^{-1}(0) g(r)
$$

is imposed for all $r$. Without loss of generality we may assume that $a(0)=b(0)=1$ and consequently we obtain a system of two first order equations which is equivalent to equation (5):

$$
\begin{aligned}
& x_{1}^{\prime}(r)-(\lambda+1) E^{-1}(r) x_{2}(r)=0 \\
& x_{2}^{\prime}(r)+[\lambda g(r)+k(r)] E(r) x_{1}(r)=0
\end{aligned}
$$

where

$$
E(r)=\exp \left[-\int_{0}^{r} f(r) d r\right]
$$

This system is defined over the interval $[0, \infty)$ with initial condition

$$
p^{-1}(0) x_{1}(0) \cos \beta+p(0) x_{2}(0) \sin \beta=0 \text {. }
$$

3. Preliminary remarks. On the finite interval $\left(0, r_{0}\right)$ let

$$
v(r, \lambda)=\left[v_{1}(r, \lambda), v_{2}(r, \lambda)\right], \quad w(r, \lambda)=\left[w_{1}(r, \lambda), w_{2}(r, \lambda)\right]
$$

be two vector solutions of (10) which satisfy the initial conditions

$$
\begin{aligned}
v_{1}(0) & =-\sin \beta & v_{2}(0) & =\cos \beta \\
w_{1}(0) & =-\cos \beta & w_{2}(0) & =-\sin \beta .
\end{aligned}
$$

The Wronskian, $W_{r}(v, w)$, is defined by the relation

$$
W_{r}(v, w)=v_{1}(r) w_{2}(r)-v_{2}(r) w_{1}(r) .
$$

From equations (10) for $v$ and $w$ we obtain 


$$
\frac{d}{d r}\left[v_{1}(r) w_{2}(r)-v_{2}(r) w_{1}(r)\right]=0
$$

which implies that $W_{r}(v, w)$ is independent of $r$. Furthermore, since $w_{0}(v, w)=1$ by (12) we may conclude that $W_{r}(v, w)=1$ for all $r$, and that $v$ and $w$ are two independent solutions of (10). Consequently a linear combination of these two independent solutions

$$
w(r, \lambda)+l(\lambda) v(r, \lambda)
$$

will be a solution of (10). It is known [2] that for Sturmian boundary conditions at $r=0$ the eigenvalues for which this solution exists will be real, nondegenerate, discrete and extend from $\lambda=-\infty$ to $\lambda=+\infty$. The corresponding eigenfunctions are real functions of $r$. For the singular case the spectrum can be investigated by considering "the limit of the solution (14) for $r \rightarrow \infty$. As in the case of singular second-order differential equations it can be shown [3] by a limit-point, limit-circle argument that for $\operatorname{Im} \lambda \neq 0$ the system (10) will have a vector solution

$$
z(r, \lambda)=w(r, \lambda)+m(\lambda) v(r, \lambda)
$$

which is $L^{2}(0, \infty)$. The function $m(\lambda)$ depends upon a limit of circles in the complex $\lambda$-plane and for $r_{0} \rightarrow \infty$ is either a limit-point or a point on a limit-circle. In the limit-circle case all solutions of system (10) are $L^{2}(0, \infty)$ and if a system is in the limit-circle case for a particular $\lambda$ it will be in the limit-circle case for all other $\lambda$ 's. Furthermore, $m(\lambda)$ is analytic for $\operatorname{Im} \lambda \neq 0$ and $m(\bar{\lambda})=\overline{m(\lambda)}$. The spectral distribution function is determined by the imaginary part of $m(\lambda)$ and the spectrum associated with a problem for which the spectral distribution function is uniquely determined is the set of non-constancy points of this distribution function. The set of all discontinuity points of the spectral distribution function comprises the point spectrum. The continuous spectrum is the set of all continuity points of this function which are in the spectrum.

4. Order properties. In order to determine the analytical character of $m(\lambda)$ we must first derive the order properties of the solutions of (10) for large values of $r$. The asymptotic behavior can be obtained by introducing a transformation [2] of the independent variable:

$$
\begin{aligned}
\alpha(r) & =\int_{0}^{r}\{(\lambda+1)[\lambda g(s)+k(s)]\}^{1 / 2} d s \\
& =\int_{0}^{r}\left\{\left(1+\lambda^{-1}\right)\left[g(s)+\lambda^{-1} k(s)\right]\right\}^{1 / 2} d s .
\end{aligned}
$$

Assume temporarily that $\lambda$ is fixed and $\operatorname{Im} \lambda>0$. The choice of a 
suitable branch cut leads to the principal value of the square root function. Here and in all other cases in which roots of complex quantities appear we will assume that the principal value of the roots have to be taken.

The transformation

$$
\begin{array}{ll} 
& r \rightarrow \alpha(r) \\
u_{1}(r)=F(r) x_{2}(r) & u_{2}(r)=G(r) x_{2}(r)-F^{-1}(r) x_{1}(r)
\end{array}
$$

yields a new system of equations:

$$
\begin{aligned}
& \frac{d u_{1}}{d \alpha}=u_{2} \\
& \frac{d u_{2}}{d \alpha}=[R(\lambda, f, g, k)-1] u_{1}
\end{aligned}
$$

where

$$
\begin{gathered}
R(\lambda, f, g, k)=G^{\prime}\left(\alpha^{\prime}\right)^{-1} F^{-1} \\
F(r, \lambda)=(1+\lambda)^{1 / 4}(\lambda g+k)^{-1 / 4} E^{-1 / 2}(r) \\
G(r, \lambda)=F^{\prime}(r, \lambda)\left(\alpha^{\prime}\right)^{-1}
\end{gathered}
$$

$R, F$ and $G$ are functions of $\lambda$ but for ease of writing we will suppress $\lambda$ in the subsequent discussion.

The reason for introducing this particular transformation is that in the new system of equations the coefficients may be bounded for large values of $r$ and $\lambda$ for cases in which the coefficients were not bounded in the old system.

The system (18) is equivalent to the system of integral equations:

$$
\begin{aligned}
\mathrm{U}_{1}(r)= & \mathrm{U}_{1}(0) \cos \alpha(r)+\mathrm{U}_{2}(0) \sin \alpha(r) \\
& +\int_{0}^{r} \mathrm{U}_{1}(s) S(s) \sin [\alpha(r)-\alpha(s)] d s \\
\mathrm{U}_{2}(r)= & -\bigcup_{1}(0) \sin \alpha(r)+\mathrm{U}_{2}(0) \cos \alpha(r) \\
& +\int_{0}^{r} \bigcup_{1}(s) S(s) \cos [\alpha(r)-\alpha(s)] d s
\end{aligned}
$$

as may be verified by substitution of $\left(\mathrm{U}_{1}, \mathrm{U}_{2}\right)$ into (18) and by defining $S(r)$ by the relation

$$
S(r)=G^{\prime}(r) F^{-1}(r) .
$$

We may state the following lemma:

LEMMA.

(i) Let $g(r)$ tend steadily to $+\infty$ and ${ }_{\mathrm{i}}^{5} g^{\prime}(r)=o\left(g^{\mu}\right)$ where 
$0<\mu<3 / 2, g^{\prime}(r) \geqq 0$ and $g^{\prime \prime}(r)$ is ultimately of one sign for $r \rightarrow \infty$.

(ii) Let $f(r)=o\left(g^{\nu}\right)$ with $\nu<0$ and $f^{\prime}(r)=o\left(g^{\omega}\right)$ with $\omega<0$ for $r \rightarrow \infty$ and let $f(r)=L(0, \infty)$.

(iii) Let $k(r)$ and its first and second derivatives be $O(1)$ for $r \rightarrow \infty$

then $\int_{0}^{\infty}|S(s)| d s$ is uuiformly bounded with respect to all $\lambda$ such that $\left|\left(1+\lambda^{-1}\right)\left(g+\lambda^{-1} k\right)\right|$ is bounded away from zero.

For ultimately there will be an $R_{0}$ such that for $r>R_{0}, S(r)$ will approach to

$$
g^{\prime \prime} g^{-3 / 2}+\left(g^{\prime}\right)^{2} g^{-5 / 2}
$$

Furthermore, since

$$
0<\mu<\frac{3}{2}
$$

we have

$$
\int_{R_{0}}^{R}\left[g^{\prime}(s)\right]^{2} g^{-5 / 2}(s) d s=O(1)
$$

and

$$
\int_{R_{0}}^{R} g^{\prime \prime}(s) g^{-3 / 2}(s) d s=O(1)
$$

The same conditions ensure that for $r \rightarrow \infty, \operatorname{Im} \alpha(r) \rightarrow+\infty$ and that $S(r), \alpha(r)$ and $F(r)$ are real for $r \rightarrow \infty$ and $\operatorname{Im} \lambda \rightarrow 0$. Consider the functions

$$
\begin{aligned}
V_{1}(r, \lambda)= & \mathrm{U}_{1}(r, \lambda) \exp [i \alpha(r)] \\
= & \exp [i \alpha(r)]\left\{\mathrm{U}_{1}(0) \cos \alpha(r)+\mathrm{U}_{2}(0) \sin \alpha(r)\right\} \\
& +\int_{0}^{r} V_{1}(s) S(s) \exp [i\{\alpha(r)-\alpha(s)\}] \sin [\alpha(r)-\alpha(s)] d s \\
V_{2}(r, \lambda)= & \mathrm{U}_{2}(r, \lambda) \exp [i \alpha(r)] \\
= & \exp [i \alpha(r)]\left\{\mathrm{U}_{2}(0) \cos \alpha(r)-\mathrm{U}_{1}(0) \sin \alpha(r)\right\} \\
& +\int_{0}^{r} V_{1}(s) S(s) \exp [i\{\alpha(r)-\alpha(s)\}] \cos [\alpha(r)-\alpha(s)] d \mathbf{s} .
\end{aligned}
$$

Applying a lemma of a previous paper [2] it can be shown that

$$
\left|V_{j}(r, \lambda)\right| \leqq\left[\left|\bigcup_{1}(0, \lambda)\right|+\left|\mathrm{U}_{2}(0, \lambda)\right|\right] \exp \left[\int_{0}^{r}|S(s)| d s\right] \quad j=1,2 .
$$

Consequently since $\int_{0}^{\infty}|S(s)| d s$ is uniformly bounded with respect to 
$\lambda$, it follows that

$$
\mathrm{U}_{j}(r, \lambda)=0\{\exp [-i \alpha(r)]\} \quad j=1,2
$$

and for a fixed $\lambda$ with $\operatorname{Im} \lambda>0$ and $r \rightarrow \infty$ we obtain:

$$
\mathrm{U}_{j}(r, \lambda)=\left[M_{j}(\lambda)+o(1)\right] \exp [-i \alpha(r)]
$$

where

$$
\begin{aligned}
& 2 M_{1}(\lambda)=\bigcup_{1}(0, \lambda)+i \bigcup_{2}(0, \lambda)+\int_{0}^{\infty} \bigcup_{1}(s) S(s) \exp [i \alpha(s)] d s \\
& 2 M_{2}(\lambda)=\bigcup_{2}(0, \lambda)-i \bigcup_{1}(0, \lambda)+\int_{0}^{\infty} \bigcup_{1}(s) S(s) \exp [i \alpha(s)] d s .
\end{aligned}
$$

For $\operatorname{Im} \lambda \rightarrow 0$ and according to (22) and (25)

$$
\begin{aligned}
& \mathrm{U}_{1}(r, \lambda)=\mu(\lambda) \sin \alpha(r)+v(\lambda) \cos \alpha(r)+o(1) \\
& \mathrm{U}_{2}(r, \lambda)=\mu(\lambda) \cos \alpha(r)-v(\lambda) \sin \alpha(r)+o(1)
\end{aligned}
$$

where

$$
\begin{aligned}
& \mu(\lambda)=\bigcup_{2}(0)+\int_{0}^{\infty} \bigcup_{1}(s) S(s) \cos \alpha(s) d s \\
& \nu(\lambda)=\bigcup_{2}(0)-\int_{0}^{\infty} \bigcup_{1}(s) S(s) \sin \alpha(s) d s
\end{aligned}
$$

and since $\int_{0}^{\infty}|S(s)| d s$ is uniformly bounded, these integrals are uniformly convergent with respect to $\lambda$ while $\mu$ and $\nu$ are continuous functions of $\lambda$. According to section (3) we have

$$
\begin{aligned}
W_{r}(v, w) & =\bigcup_{1 v} \bigcup_{2 w}-\bigcup_{2 v} \bigcup_{1 w} \\
& =\mu_{w} \nu_{v}-\mu_{v} \nu_{w}+o(1)=1
\end{aligned}
$$

and for $r \rightarrow \infty$

$$
\mu_{w} \nu_{v}-\mu_{v} \nu_{w}=1
$$

For large values of $|\lambda|$ and fixed finite $r$ we obtain

$$
\alpha(r)=\lambda \Phi(r)+\Psi(r)+o\left(\lambda^{-1}\right)
$$

where

$$
\Phi(r)=\int_{0}^{r}[g(s)]^{1 / 2} d s
$$

and

$$
\Psi(r)=\frac{1}{2} \int_{0}^{r}[k(s)+g(s)][g(s)]^{-1 / 2} d s .
$$


If we define $p(r)$ by the relation

$$
p(r)=E^{-1 / 2}(r)[g(r)]^{-1 / 4}
$$

then

$$
\begin{aligned}
& F(r)=p(r)+O\left(\lambda^{-1}\right) \\
& G(r)=O\left(\lambda^{-1}\right), \quad S(r)=O\left(\lambda^{-1}\right) .
\end{aligned}
$$

Hence

$$
\begin{aligned}
& \mathrm{U}_{1}(0)=\left[p(0)+O\left(\lambda^{-1}\right)\right] x_{2}(0) \\
& \bigcup_{2}(0)=\left[-p^{-1}(0)+O\left(\lambda^{-1}\right)\right] x_{1}(0)+x_{2}(0) O\left(\lambda^{-1}\right) .
\end{aligned}
$$

A similar argument as the one presented above shows that for large $|\lambda|$ :

$$
x(r) \equiv x_{1}(r)=p(r) \sin [\alpha(r)-\beta]+O\left\{[\exp |\tau| \Phi(r)]|\lambda|^{-1}\right\}
$$

where $\tau=\operatorname{Im} \lambda$.

5. A spectral theorem. We are now in a position to proof the following spectral theorem for the system of equations (10).

THEOREM. Let $f, g$ and $k$ satisfy the conditions imposed in Lemma 1. The system (10) with initial conditions (11) will have a. two component vector solution $\vec{x}=\left(x_{1}, x_{2}\right)$ over the interval $[0, \infty)$ and the values of $\lambda$ for which such a solution exist are in the continuous spectrum.

As was stated in section (3) the spectral properties of system (10). depend on the analytic character of $m(\lambda)$ as a function of the complex variable $\lambda$ in the limit for $\operatorname{Im} \lambda \rightarrow 0$. From Weyl's limit-point, limitcircle theorem it follows that for $\operatorname{Im} \lambda \neq 0$ (10) always will have at least one two component vector solution

$$
z(r, \lambda)=w(r, \lambda)+m(\lambda) v(r, \lambda)
$$

which is in $L^{2}(0, \infty)$. For $r \rightarrow \infty, \operatorname{Im} \alpha(r) \rightarrow \infty$ and

$$
\begin{aligned}
z_{1}= & w_{1}(r, \lambda)+m(\lambda) v_{1}(r, \lambda) \\
= & \left\{G(\lambda) M_{1 w}(\lambda)-F(\lambda) M_{2 w}(\lambda)+m(\lambda)\left\{G(\lambda) M_{1 v}(\lambda)-F(\lambda) M_{2 v}(\lambda)\right\}\right. \\
& +o(1)\} \exp [-i \alpha(r)] \\
z_{2}= & w_{2}(r, \lambda)+m(\lambda) v_{2}(r, \lambda) \\
= & {\left[F^{-1}(\lambda) M_{1 w}(\lambda)+m(\lambda) F(\lambda) M_{1 v}(\lambda)+o(1)\right] \exp [-i \alpha(r)] . }
\end{aligned}
$$

Hence if $z$ is to be in $L^{2}(0, \infty)$ 


$$
m(\lambda)=-\frac{M_{1 w}(\lambda)}{M_{2 v}(\lambda)}=-\lim _{r \rightarrow \infty} \frac{G(r, \lambda) M_{1 w}(\lambda)-F(r, \lambda) M_{2 w}(\lambda)}{G(r, \lambda) M_{1 v}(\lambda)-F(r, \lambda) M_{2 v}(\lambda)}
$$

but by (25) for $r \rightarrow \infty$

$$
2 M_{1 v}(\lambda)=\bigcup_{1 v}(0, \lambda)+i \bigcup_{2 v}(0, \lambda)+i \int_{0}^{\infty} e^{i \omega(s)} S(s) F(s) v_{2}(s) d s
$$

and

$$
\begin{aligned}
& \bigcup_{2 v}(0, \lambda)=F^{-1}(0, \lambda) \sin \beta+G(0, \lambda) \cos \beta \\
& \bigcup_{1 v}(0, \lambda)=F(0, \lambda) \cos \beta .
\end{aligned}
$$

Hence

$$
\begin{aligned}
& 2 M_{1 v}(\lambda)=v_{v}(\lambda)+i \mu_{v}(\lambda) \\
& 2 M_{2 v}(\lambda)=\mu_{v}(\lambda)-i v_{v}(\lambda) \\
& 2 M_{1 w}(\lambda)=v_{w}(\lambda)+i \mu_{w}(\lambda) \\
& 2 M_{2 w}(\lambda)=\mu_{w}(\lambda)-i v_{w}(\lambda)
\end{aligned}
$$

and

$$
-m(\lambda)=\frac{\mu_{w} \mu_{v}+v_{w} \nu_{v}}{\mu_{v}^{2}+v_{v}^{2}}+\frac{i}{\mu_{v}^{2}+v_{v}^{2}}
$$

and

$$
-\operatorname{Im} m(\lambda)=\frac{1}{\mu_{v}^{2}+\nu_{v}^{2}} .
$$

Since, by (26) $\mu_{v}$ and $v_{v}$ cannot vanish simultaneously, this shows that $\operatorname{Im} m(\lambda)$ is a continuous non vanishing function of $\lambda$. Consequently the spectral distribution function is continuous and $\lambda$ will be in the continuous spectrum [6]. The results of this section can now immediately be applied to obtain similar statements with respect to the spectral properties of equation (5).

APPENDIX. The example referred to in section (1)

$$
\text { (A1) } u^{\prime \prime}+2 r^{-1} u^{\prime}+\left[\lambda^{2}+2 \lambda e V(r)+e^{2} V^{2}(r)-l(l+1) r^{-2}-1\right] u=0
$$

does not satisfy the conditions of the theorem. Of course, it is always possible to reduce equation (A1) into a system of equations. For instance, for a Coulomb potential

$$
V(r)=e Z r^{-1}
$$

the following system of equations seems suitable

$$
\begin{aligned}
& x_{1}^{\prime}(r)-\lambda a(r) x_{2}(r)=0 \\
& x_{2}^{\prime}(r)+[\lambda c(r)+\alpha(r)] x_{1}(r)=0 .
\end{aligned}
$$


In this case we obtain the auxiliary equations

$$
\begin{gathered}
a(r) c(r)=1 \\
a(r) d(r)=2 c^{2} Z r^{-1} \\
\frac{1}{2} \frac{d}{d \lambda}\left(\frac{a^{\prime}}{a}\right)-\frac{1}{4}\left(\frac{a^{\prime}}{a}\right)^{2}=\frac{e^{4} Z}{r^{2}}-\frac{l(l+1)}{r^{2}}-1 .
\end{gathered}
$$

For

$$
a^{\prime} / a=-2 u^{\prime} / u
$$

we obtain

$$
u^{\prime \prime}+\left[-1+\frac{c^{4} Z^{2}-l(l+1)}{r^{2}}\right] u=0 .
$$

The solution of this equation which is finite at $r \rightarrow \infty$ is a spherical Hankel function of imaginary argument and complex order

$$
u(r)=r h_{i p-1 / 2}(i r)
$$

where

$$
p=\left[e^{4} Z^{2}-l(l+1)-1 / 4\right]^{1 / 2} .
$$

For $r \rightarrow \infty$

$$
u(r) \rightarrow-i \exp \left[-r-\frac{1}{2} i \pi\left(i p+\frac{1}{2}\right)\right]
$$

and it may be verified that for this substitution $S(r)$ is not $L(0, \infty)$ which would render the above approach invalid. This same obstacle was found for the Dirac equation for an electron in a Coulomb field [3]. Fortunately these cases may be solved exactly and the difficulties encountered are purely academic.

\section{REFERENCES}

1. K. Case, Singular potentials, Phs. Rev., 80 (1950), 797-806.

2. B. W. Roos and W. C. Sangren, Proc. Amer Math. Soc., 12, (1961), 468-476.

3. - Journal of Mathematical Physics, 3 (1962), 882-890.

4. —, Pacific J. Math., 12 (1962) 1047-1055.

5. E. C. Titchmarsh, Eigen function Expansions associated with second order differential equations, Oxford, Clarendon Press (1962).

6. — Proc. London Math. Soc., (3) 11 (1961), 159-168.

7. H. Weyl, Math. Ann., 68 (1910), 220-269.

General Atomic Division of General Dynamics Corporation John Jay Hopkins Laboratory, San Diego, California 


\section{PACIFIC JOURNAL OF MATHEMATICS}

\section{EDITORS}

Robert Osserman

Stanford University

Stanford, California

M. G. Arsove

University of Washington

Seattle 5 , Washington
J. DugundjI

University of Southern Califorma: Los Angeles 7, California

Lowell J. Paige

University of California

Los Angeles 24, California

\section{ASSOCIATE EDITORS}

E. F. BECKENBACH

B. H. NeumanN

F. WOLF

K. YosIDA

\section{SUPPORTING INSTITUTIONS}

UNIVERSITY OF BRITISH COLUMBIA

CALIFORNIA INSTITUTE OF TECHNOLOGY

UNIVERSITY OF CALIFORNIA

MONTANA STATE UNIVERSITY

UNIVERSITY OF NEVADA

NEW MEXICO STATE UNIVERSITY

OREGON STATE UNIVERSITY

UNIVERSITY OF OREGON

OSAKA UNIVERSITY

UNIVERSITY OF SOUTHERN CALIFORNIA
STANFORD UNIVERSITY

UNIVERSITY OF TOKYO

UNIVERSITY OF UTAH

WASHINGTON STATE UNIVERSITY

UNIVERSITY OF WASHINGTON

AMERICAN MATHEMATICAL SOCIETY CALIFORNIA RESEARCH CORPORATION SPACE TECHNOLOGY LABORATORIES NAVAL ORDNANCE TEST STATION 


\section{Pacific Journal of Mathematics}

\section{Vol. 14, No. 1 \\ May, 1964}

Richard Arens, Normal form for a Pfaffian .........................

Charles Vernon Coffman, Non-linear differential equations on cones in Banach

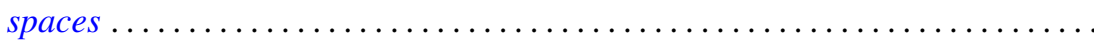

Ralph DeMarr, Order convergence in linear topological spaces ..............

Peter Larkin Duren, On the spectrum of a Toeplitz operator ................

Robert E. Edwards, Endomorphisms of function-spaces which leave stable all

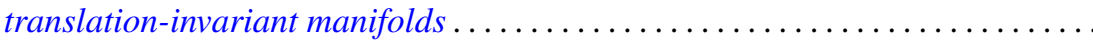

Erik Maurice Ellentuck, Infinite products of isols . . . . . . . . . . . . . . . . 49

William James Firey, Some applications of means of convex bodies . . . . . . . . 53

Haim Gaifman, Concerning measures on Boolean algebras ............. 61

Richard Carl Gilbert, Extremal spectral functions of a symmetric operator. . . . . . 75

Ronald Lewis Graham, On finite sums of reciprocals of distinct nth powers ..... 85

Hwa Suk Hahn, On the relative growth of differences of partition functions ...... 93

Isidore Isaac Hirschman, Jr., Extreme eigen values of Toeplitz forms associated

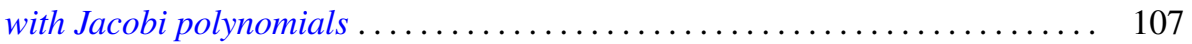

Chen-jung Hsu, Remarks on certain almost product spaces . . . . . . . . . . . 163

George Seth Innis, Jr., Some reproducing kernels for the unit disk . . . . . . . . . 177

Ronald Jacobowitz, Multiplicativity of the local Hilbert symbol . . . . . . . . . . . 187

Paul Joseph Kelly, On some mappings related to graphs ................. 191

William A. Kirk, On curvature of a metric space at a point . . . . . . . . . . . . 195

G. J. Kurowski, On the convergence of semi-discrete analytic functions . . . . . . . 199

Richard George Laatsch, Extensions of subadditive functions . . . . . . . . . . . 209

V. Marić, On some properties of solutions of $\Delta \psi+A\left(r^{2}\right) X \nabla \psi+C\left(r^{2}\right) \psi=0 \ldots 217$

William H. Mills, Polynomials with minimal value sets . . . . . . . . . . . 225

George James Minty, Jr., On the monotonicity of the gradient of a convex

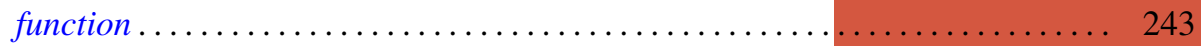

George James Minty, Jr., On the solvability of nonlinear functional equations of 'monotonic' type ................................... 249

J. B. Muskat, On the solvability of $x^{e} \equiv e(\bmod p) \ldots \ldots \ldots \ldots \ldots \ldots \ldots \ldots . \ldots \ldots$

Zeev Nehari, On an inequality of $P . R$. Bessack ................... 261

Raymond Moos Redheffer and Ernst Gabor Straus, Degenerate elliptic

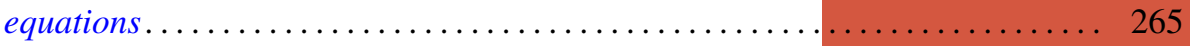

Abraham Robinson, On generalized limits and linear functionals . . . . . . . . . 269

Bernard W. Roos, On a class of singular second order differential equations with a

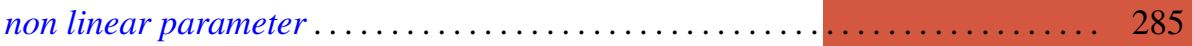

Tôru Saitô, Ordered completely regular semigroups . . . . . . . . . . . . . . . . 295

Edward Silverman, A problem of least area ....................... 309

Robert C. Sine, Spectral decomposition of a class of operators . . . . . . . . . 333

Jonathan Dean Swift, Chains and graphs of Ostrom planes . . . . . . . . . . . 353

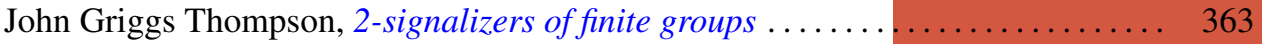

Harold Widom, On the spectrum of a Toeplitz operator . . . . . . . . . . . . . 365 